\title{
RESEARCH HIGHLIGHT Young haematopoietic stem cells are picky eaters
}

\author{
Nick van Gastel $\mathbb{D}^{1,2}$ and David T. Scadden ${ }^{1,3}$ \\ Cell Research (2021) 31:377-378; https://doi.org/10.1038/s41422-021-00488-8
}

\begin{abstract}
In a recent study published in Nature, Dong et al. describe how haematopoietic stem cells (HSCs) rely on chaperonemediated autophagy (CMA), a form of highly selective lysosomal protein degradation, as a metabolic quality control mechanism. While old HSCs have reduced CMA activity, reactivation of CMA can boost their functionality, providing exciting therapeutic perspectives.
\end{abstract}

Blood cell production arises from the activity of haematopoietic stem cells (HSCs), defined by their capacity to differentiate into myeloid and lymphoid lineages and self-renew. These cell fate decisions must be carefully balanced to allow blood cell production and meet the shifting demands of the immune system, while maintaining a sufficient pool of functional HSCs. Central to preserving HSC functionality and survival is the dynamic control of their metabolic state. Under homeostatic conditions, HSCs rely on glycolysis - the conversion of glucose to pyruvate, and fermentation - the conversion of pyruvate to lactate, in order to maintain their quiescent state. ${ }^{1,2}$ When activated, HSCs engage mitochondrial metabolism, fueled predominantly by fatty acid oxidation. ${ }^{3}$ To preserve self-renewal during division, HSCs rely on mitochondrial quality control mechanisms including the mitochondrial unfolded protein response ${ }^{4}$ mitochondrial oxidative stress mitigation ${ }^{5}$ and autophagic degradation of damaged mitochondria, called mitophagy.

With ageing, HSCs undergo metabolic reprogramming characterized by increased mitochondrial oxidative phosphorylation, accumulation of reactive oxygen species (ROS) and reduced efficacy of metabolic quality control, thought to compromise HSC function. ${ }^{7}$ Accordingly, induction of metabolic perturbations can induce premature HSC ageing. Aged HSCs display several distinct phenotypes, including an increase in frequency, reduction of regenerative capacity, and a myeloid differentiation bias. ${ }^{8}$ With the promise of discovering ways to prevent HSC ageing or restore old HSC function, the search for mechanisms that keep HSCs young has intensified in recent years.

A new study from the groups of Ana Maria Cuervo and Britta Will at the Albert Einstein College of Medicine in New York has identified chaperone-mediated autophagy (CMA) as an essential metabolic quality control mechanism in $\mathrm{HSCs}^{9}$ CMA is a highly selective form of autophagy in which proteins bearing a targeting motif (KFERQ-like) are recognized by the heat shock cognate protein $\mathrm{HSC70}$ and are targeted to the lysosomes for degradation, a process involving binding to the lysosome-associated membrane protein LAMP2A. ${ }^{10}$ Using a CMA reporter mouse strain (KFERQ-Dendra2), the authors showed that quiescent HSCs from young mice had high basal CMA activity compared to myeloid progenitors, and CMA was further upregulated when HSCs were activated in vivo with the myeloablative agent 5 -fluorouracil
(5-FU). Old HSCs in contrast showed lower CMA activity on a per cell basis and a lower fraction of HSCs active for CMA when compared to young HSCs.

Deletion of Lamp2a in the hematopoietic system did not impact mature blood cell numbers, but reduced the number of HSCs in the bone marrow and compromised their ability to expand in response to 5-FU or to reconstitute the hematopoietic system after sequential transplantation. HSCs with defective CMA showed delayed cycling and limited self-renewal, linked to low ATP levels and accumulation of ROS. In line with this, loss of LAMP2A in HSCs led to the accumulation of proteins involved in metabolism, many of which were identified to be putative CMA substrates. Elegant metabolic studies revealed impaired glycolysis in CMA-defective HSCs, as shown by the accumulation of oxidized forms of the key glycolytic enzymes glyceraldehyde-3-phosphate dehydrogenase and pyruvate kinase, both of which are known CMA substrates. Activation of HSCs by $5-\mathrm{FU}$ revealed additional metabolic roles for CMA, including the regulation of fatty acid metabolism. The authors identified the fatty acid desaturase FADS2 as a key CMA target in activated HSCs, where selective degradation of inactive, acetylated FADS2 is required to maintain flux through linoleic and a-linolenic metabolism in support of fatty acid oxidation.

Several of the defects found in young CMA-defective HSCs were remarkably similar to those found in HSCs derived from aged control mice, including proteome changes, defective glycolysis and reduced flux through linoleic acid metabolism, in line with the observation that old HSCs have reduced CMA activity. Interestingly, only about half of old HSCs showed defective CMA activity, while the other half was nearly indistinguishable from young HSCs. Restoration of CMA in old HSCs, either through LAMP2A overexpression or oral administration of a pharmacological CMA activator to old mice, reduced oxidized protein levels and restored activity of both glycolysis and linoleic acid metabolism, leading to improved HSC function. Similarly, treatment of $\mathrm{CD}_{3} 4^{+}$human haematopoietic stem and progenitor cells from old donors with the CMA activator markedly increased the number of functional stem cells in long-term culture, supporting the idea that pharmacological activation of CMA could be effective in improving and restoring aged HSC function.

The very comprehensive study by Dong et al. presents a strong case for the essential role of CMA in the control of HSC fate and function, especially through the regulation of glucose and fatty acid metabolism. In addition, several new questions are raised by the findings of this study. First, the heterogeneity of CMA activity in old HSCs is remarkable, and future studies should be designed to decipher the differences between these two subpopulations of old HSCs and the mechanisms behind the loss of CMA activity in

\footnotetext{
${ }^{1}$ Department of Stem Cell and Regenerative Biology, Harvard Stem Cell Institute, Harvard University, Cambridge, MA 02138, USA; ${ }^{2}$ de Duve Institute, Brussels, Belgium and ${ }^{3}$ Center for Regenerative Medicine, Massachusetts General Hospital, Boston, MA 02138, USA

Correspondence: David T. Scadden (david_scadden@harvard.edu)
}

Published online: 25 February 2021 
some HSCs with ageing. Second, since CMA, similar to other types of autophagy, is often activated in response to nutrient stress, ${ }^{10}$ an investigation into the relative roles of CMA and macroautophagy in HSCs during periods of starvation or in response to excess caloric intake would be very relevant. Finally, several other metabolic pathways were identified as changed in CMAdefective HSCs, and it will be of considerable interest to further dissect the role of CMA in the regulation of metabolic homeostasis during HSC quiescence as well as metabolic rewiring upon HSC activation.

Once again, this study highlights the importance of metabolic programs and quality control systems in the regulation of stem cell fate and function. The ability of young HSCs to selectively remove damaged metabolic enzymes may allow them to meet metabolic demands with high efficiency and great flexibility, avoiding the build-up of unnecessary metabolites or toxic byproducts that can compromise stem cell function.

\section{ADDITIONAL INFORMATION}

Competing interests: D.T.S. is a director and equity holder of Agios Pharmaceuticals, Magenta Therapeutics, Editas Medicines, Clear Creek Bio and LifeVaultBio; he is a founder of Fate Therapeutics and Magenta Therapeutics and a consultant to FOG Pharma, VCanBio and Flagship Pioneering. N.v.G. has no competing interests to declare.

\section{REFERENCES}

1. Takubo, K. et al. Cell Stem Cell 12, 49-61 (2013).

2. Wang, Y. H. et al. Cell 158, 1309-1323 (2014).

3. Ito, K. et al. Nat. Med. 18, 1350-1358 (2012).

4. Mohrin, M. et al. Science 347, 1374-1377 (2015).

5. Ito, K. et al. Nat. Med. 12, 446-451 (2006).

6. Ito, K. et al. Science 354, 1156-1160 (2016).

7. Nakamura-Ishizu, A., Ito, K. \& Suda, T. Dev. Cell 54, 239-255 (2020).

8. Geiger, H., de Haan, G. \& Florian, M. C. Nat. Rev. Immunol. 13, 376-389 (2013).

9. Dong, S. et al. Nature https://doi.org/10.1038/s41586-020-03129-z (2021).

10. Kaushik, S. \& Cuervo, A. M. Nat. Rev. Mol. Cell Biol. 19, 365-381 (2018). 